\title{
Time-to-operation does not predict outcome in acute type A aortic dissection complicated by neurologic injury at presentation
}

Peter Chiu, MD, MS, Torsten J. Rotto, BA, Andrew B. Goldstone, MD, PhD, Joshua B. Whisenant, Y. Joseph Woo, MD, and Michael P. Fischbein, MD, PhD

\section{ABSTRACT}

Objective: Neurologic injury complicating the presentation of acute type A aortic dissection remains a challenge for cardiac surgeons.

Methods: This was a retrospective review of patients undergoing open repair of acute type A aortic dissection at our institution between January 2005 and December 2015. Evidence of neurologic injury at the time of presentation was abstracted from the medical record. Propensity-score matching was used to account for baseline differences between groups, and outcome analysis was performed using logistic regression and Kaplan-Meier analysis. Among patients with persistent neurologic deficits, a threshold for time-to-operation was evaluated using receiver operating characteristic curves.

Results: There were 345 patients who underwent open repair for acute type A aortic dissection; 50 patients presented with neurologic injury. In the matched analysis, in-hospital mortality was greater among patients who presented with neurologic deficits (odds ratio, 4.42; 95\% confidence interval, 1.15-16.97; $P=.03$ ). Among patients with persistent neurologic deficits at presentation, receiver operating characteristic curve analysis with cross-validation suggested that time-to-operation was a poor predictor of both neurologic outcome (area under the curve, 0.40) and death (area under the curve, 0.49).

Conclusions: Neurologic injury at the time of presentation with acute type A aortic dissection was associated with an increased risk of in-hospital mortality. Among patients with persistent neurological deficits, time-to-operation failed to predict either neurologic outcome or perioperative mortality suggesting that longer time from onset of symptoms of neurologic injury should not act as a contraindication to proceeding to the operating room for expedient repair. ( $\mathrm{J}$ Thorac Cardiovasc Surg 2019;158:665-72)

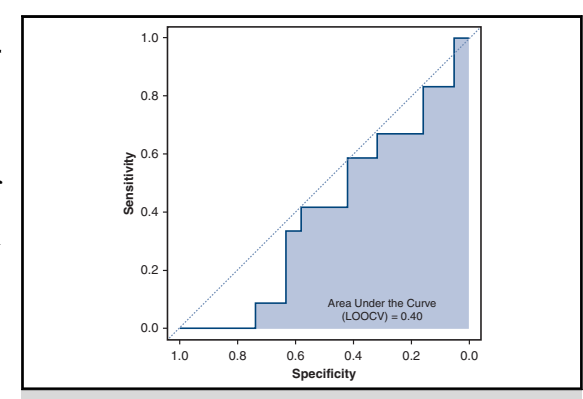

Increasing time-to-operation was a poor predictor of lack of neurologic improvement.

\section{Central Message}

Among patients with acute type $\mathrm{A}$ aortic dissection complicated by neurologic injury, time-to-operation does not predict either neurologic outcome or death. Expedient repair should be undertaken.

\section{Perspective}

Acute type A aortic dissection complicated by neurologic injury remains a clinical challenge for cardiovascular surgeons. Among patients presenting with persistent neurologic deficits, time-to-operation failed to predict either neurologic outcome or death, suggesting that longer time from onset of neurologic symptoms should not act as a contraindication to proceeding with immediate operation.

See Commentary on page 673 .
From the Department of Cardiothoracic Surgery, School of Medicine, Stanford University, Stanford, Calif.

Dr Peter Chiu was supported by a KL2 Mentored Career Development Award of the Stanford Clinical and Translational Science Award to Spectrum (National Institutes of Health grant No. KL2 TR 001083).

Received for publication June 5, 2018; revisions received Dec 2, 2018; accepted for publication Dec 7, 2018; available ahead of print Feb 1, 2019.

Address for reprints: Michael P. Fischbein, MD, PhD, Department of Cardiothoracic Surgery, School of Medicine, Stanford University, Falk CVRB, 300 Pasteur Dr, Falk CVRB ULN MC5407, Stanford, CA 94305 (E-mail: mfischbe@stanford. edu).

0022-5223

Copyright (C) 2018 by The American Association for Thoracic Surgery. Published by Elsevier Inc. This is an open access article under the CC BY-NC-ND license (http:// creativecommons.org/licenses/by-nc-nd/4.0/).

https://doi.org/10.1016/j.jtcvs.2018.12.023
Acute type A aortic dissection is a surgical emergency requiring immediate operation. ${ }^{1}$ Prior reports have suggested that neurologic injury may be a contraindication to surgical therapy given concerns about hemorrhagic complications associated with systemic heparinization for cardiopulmonary bypass in addition to the high incidence of perioperative morbidity and mortality. ${ }^{2-4}$ In the International Registry for Acute Aortic Dissection, 33\% of patients presenting with coma and $24.1 \%$ of patients presenting with cerebrovascular accident underwent nonoperative management with $100 \%$ and $76.2 \%$ inhospital mortality, respectively. ${ }^{5}$ 


\section{Abbreviations and Acronyms \\ AUC $=$ area under the curve \\ GCS $=$ Glasgow Coma Scale}

Despite the risks, neurologic injury has not been used as a reason to preclude surgery at Stanford. ${ }^{6}$ In line with this approach, Estrera and colleagues ${ }^{7}$ reported excellent outcomes in a study of 14 patients who underwent immediate operation for acute type A aortic dissection complicated by stroke. Tsukube and colleagues ${ }^{8}$ subsequently reported on the outcome of immediate operative repair for patients with acute type A aortic dissection complicated by coma in 21 patients. Both studies emphasized the timeliness of operative intervention with outcomes declining precipitously beyond 10 hours in the study by Estrera and colleagues ${ }^{7}$ and 5 hours in the study by Tsukube and colleagues. ${ }^{8}$ Until recently, this timeframe was consistent with the existing evidence suggesting that intervention in acute ischemic stroke might have limited benefit beyond 6 to 7 hours. $^{9-11}$ This time limit has recently been challenged in the stroke literature with advances in mechanical thrombectomy. ${ }^{12-14}$ Considering the evolving understanding of acute ischemic stroke and the limited published data on acute type A aortic dissection complicated by neurologic injury, whether the upper limit of time-to-operation has been delineated remains unclear. We undertook the current study to evaluate the outcome of immediate unselected operative therapy for patients with neurologic injury complicating the presentation of acute type A aortic dissection.

\section{METHODS}

This was a retrospective review of patients treated for acute type A aortic dissection at Stanford Healthcare between January 1, 2005, and December 31, 2015. Following approval from the Stanford University Institutional Review Board, we identified patients using departmental databases and a query of billing data in the Stanford Translational Research Integrated Database Environment. Patients treated nonoperatively and patients undergoing primary endovascular repair were excluded.

Patients with neurologic injuries were identified by reviewing the medical record, including documentation from both referring hospitals and medical transportation services. Patients were classified as having a persistent preoperative neurologic injury if the deficit was present at the time of operation. In the case that the deficit had resolved, the patient was classified as having a transient preoperative neurologic injury. Neurologic injury was defined as obtundation/coma, verbal component of the Glasgow Coma Scale (GCS) $<5$, or focal neurologic deficits (eg, facial droop or hemiplegia) without evidence of malperfusion.

\section{Outcomes}

The primary end point was perioperative mortality as defined by death before discharge. Midterm survival was additionally analyzed as a secondary end point. Use of the Social Security Death Index is complicated by significant missingness, making it unusable as the sole source of follow-up data. ${ }^{15}$ As a result, we used a combination of the Social Security Death
Index, integration of the electronic medical record with regional health systems, and direct patient contact. Our protocol for follow-up involves a dedicated group of advanced practice practitioners, surgeons, radiologists, and administrative assistants and is similar to the process reported by Peterss and colleagues. ${ }^{16}$ Patients were censored at the time of last follow-up.

For our evaluation of a threshold for time-to-operation, both perioperative mortality and improvement of symptoms with or without complete resolution of neurologic deficit were used as outcomes. Patients were considered improved if there was a persistent deficit following the operation that was less severe than before the operation; that is, if the patient presented hemiplegic but was discharged with only weakness of one limb. Resolution was defined as having no evidence of focal neurological deficit at discharge. Improvement and resolution were determined by review of medical records, including consultation reports from neurology, physical and occupational therapy, and speech and language pathology.

\section{Statistical Analysis}

Continuous variables are presented with standard deviation or interquartile range, for non-normally distributed data. Comparisons were made with Student $t$ test or Wilcoxon rank-sum test, as appropriate. Categorical variables are reported with absolute counts and percentages, and comparisons were made with the $\chi^{2}$ test or Fisher exact test, as necessary. Odds ratios (ORs) are presented with $95 \%$ confidence interval (CI). Due to the exploratory nature of this study, no adjustment was made for multiple comparisons. ${ }^{17}$

To account for baseline differences in patients with and without neurologic injuries at the time of presentation, we used propensity-score matching. The probability of a neurologic injury at presentation was estimated using nonparsimonious logistic regression. Patients were then matched using 1:1 optimal matching without a caliper (optmatch package). Balance was assessed using the standardized mean differences approach, and a standardized mean difference $<0.2$ was considered adequate. ${ }^{18}$ We then used logistic regression in our matched cohort to evaluate the effect of neurologic injury on perioperative mortality. We planned to interrogate the different presenting symptoms in an exploratory analysis should a statistically significant difference arise between patients with and without neurologic injury at presentation. Midterm survival-our secondary outcomewas assessed using the Kaplan-Meier method and the log-rank test with the survival package. All analyses were performed in R version 3.4.1 (R Foundation for Statistical Computing, Vienna, Austria).

\section{Extent of Neurologic Injury and Postoperative Outcome}

An exploratory analysis was performed to evaluate whether the primary presenting symptoms influenced perioperative mortality. Symptoms were separated into persistent or transient injuries; of patients with persistent neurologic injuries, the primary presenting symptoms were further classified as obtunded/coma, altered mental status (verbal component of GCS $<5$ but not obtunded), hemiplegia, or other neurologic injury. Stepwise selection methods are prone to producing irreproducible models ${ }^{19,20}$; bootstrap techniques may similarly be prone to these issues. ${ }^{21}$ We used the screen-and-clean approach to variable selection in high-dimensional feature spaces to construct a sparse model and limit the possibility of overfitting. ${ }^{22,23}$ Least absolute shrinkage and selection operator regression with cross-validation for selection of the lambda penalty coefficient was used to screen potential covariates and hypothesis testing was employed to clean the remaining covariates retaining only those variables that were statistically significant. $^{22,23}$

\section{Time-to-Operation and Neurologic Outcome}

Given the time-dependence of outcome reported by Estrera and colleagues ${ }^{7}$ and Tsukube and colleagues ${ }^{8}$ we explored the effect of time-tooperation on postoperative outcome. Receiver operating characteristic 
curves were employed to determine the discrimination ability of a time-tooperation threshold for postoperative neurologic improvement and perioperative mortality; leave-one-out cross-validation was employed to reduce the optimism of the area under the curve (AUC) estimate. An AUC of 0.50 suggests that the predictor is no better than chance.

\section{RESULTS}

There were 391 patients with acute type A aortic dissection who presented to Stanford Healthcare between January 2005 and December 2015. There were 31 patients $(7.9 \%)$ who were treated nonoperatively and thus excluded. Of these, 3 patients presented with persistent neurologic injuries: 1 patient had died by drowning and was deemed inoperable, 1 patient arrived in irreversible shock with multiorgan system failure on high-dose epinephrine infusion and evidence of disseminated intravascular coagulation and was deemed inoperable, and 1 patient arrived with altered mental status and the family declined surgery on account of an existing do not resuscitate order. An additional 15 patients $(3.8 \%)$ were treated endovascularly and excluded. Of these patients, only 1 presented with a persistent neurological deficit (obtundation); this patient was treated with endovascular repair on account of a primary intimal tear in the descending thoracic aorta.

There were 345 patients remaining who underwent open surgical repair for acute type A aortic dissection, and 50 patients $(14.5 \%)$ presented with neurologic injuries. Of these patients, $62 \%$ (31 of 50) had persistent neurologic injuries at presentation, and 38\% (19 of 50) experienced transient neurologic injuries; that is, the deficit had resolved before proceeding to the operating room. Before matching, the group with neurologic injuries at presentation had a lower frequency of comorbid diabetes, dyslipidemia, dialysis, or Marfan syndrome. These patients more commonly presented with shock and distal malperfusion syndromes (eg, limb, renal, or mesenteric malperfusion) but less commonly

TABLE 1. Cohort before and after matching. Before matching, limited differences in comorbidities existed. Propensity-score matching adequately adjusted for these baseline differences

\begin{tabular}{|c|c|c|c|c|c|c|}
\hline \multirow[b]{2}{*}{ Variable } & \multicolumn{2}{|c|}{ Before matching } & \multirow[b]{2}{*}{ SMD } & \multicolumn{2}{|c|}{ After matching } & \multirow[b]{2}{*}{ SMD } \\
\hline & $\begin{array}{c}\text { No } \\
\text { neurologic } \\
\text { injury }\end{array}$ & $\begin{array}{c}\begin{array}{c}\text { Neurologic } \\
\text { injury }\end{array} \\
(\mathbf{n}=\mathbf{5 0})\end{array}$ & & 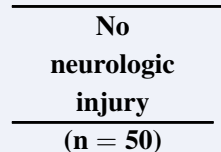 & $\begin{array}{c}\begin{array}{c}\text { Neurologic } \\
\text { injury }\end{array} \\
(\mathbf{n}=\mathbf{5 0})\end{array}$ & \\
\hline \multicolumn{7}{|l|}{ Patient demographic characteristic } \\
\hline Operative year & $2011(2008-2014)$ & $2011(2008.25-2013.75)$ & 0.059 & $2012(2009-2014)$ & $2011(2008.25-2013.75)$ & 0.079 \\
\hline Age $(y)$ & $59.06 \pm 13.73$ & $57.84 \pm 12.81$ & 0.092 & $57.59 \pm 10.83$ & $57.84 \pm 12.81$ & 0.021 \\
\hline Female & $86(29.2)$ & $13(26)$ & 0.071 & $15(30)$ & $13(26)$ & 0.089 \\
\hline White & $160(54.2)$ & $27(54)$ & 0.005 & $26(52)$ & $27(54)$ & 0.040 \\
\hline Black & $19(6.4)$ & $3(6)$ & 0.018 & $4(8)$ & $3(6)$ & 0.078 \\
\hline \multicolumn{7}{|l|}{ Comorbid conditions } \\
\hline Prior stroke & $9(3.1)$ & $3(6)$ & 0.142 & $3(6)$ & $3(6)$ & $<0.001$ \\
\hline Heart failure & $18(6.1)$ & $2(4)$ & 0.096 & $2(4)$ & $2(4)$ & $<0.001$ \\
\hline Coronary artery disease & $32(10.8)$ & $3(6)$ & 0.175 & $2(4)$ & $3(6)$ & 0.092 \\
\hline Atrial fibrillation & $26(8.8)$ & $3(6)$ & 0.108 & $3(6)$ & $3(6)$ & $<0.001$ \\
\hline Hypertension & $257(87.1)$ & $42(84)$ & 0.089 & $42(84)$ & $42(84)$ & $<0.001$ \\
\hline Chronic obstructive pulmonary disease & $38(12.9)$ & $4(8)$ & 0.160 & $6(12)$ & $4(8)$ & 0.134 \\
\hline Diabetes & $19(6.4)$ & $1(2)$ & 0.222 & $1(2)$ & $1(2)$ & $<0.001$ \\
\hline Hyperlipidemia & $83(28.1)$ & $9(18)$ & 0.242 & $13(26)$ & $9(18)$ & 0.194 \\
\hline Dialysis & $7(2.4)$ & $0(0)$ & 0.220 & $50(100)$ & $50(100)$ & $<0.001$ \\
\hline Chronic renal insufficiency & $50(16.9)$ & $5(10)$ & 0.205 & $5(10)$ & $5(10)$ & $<0.001$ \\
\hline Bicuspid & $17(5.8)$ & $4(8)$ & 0.088 & $3(6)$ & $4(8)$ & 0.078 \\
\hline Marfan syndrome & $13(4.4)$ & $0(0)$ & 0.304 & $50(100)$ & $50(100)$ & $<0.001$ \\
\hline Redo sternotomy & $17(5.8)$ & $3(6)$ & 0.010 & $3(6)$ & $3(6)$ & $<0.001$ \\
\hline \multicolumn{7}{|l|}{ Clinical characteristics } \\
\hline Shock & $34(11.5)$ & $12(24)$ & 0.331 & $9(18)$ & $12(24)$ & 0.148 \\
\hline Distal malperfusion & $63(21.4)$ & $20(40)$ & 0.413 & $22(44)$ & $20(40)$ & 0.081 \\
\hline Myocardial infarction & $8(2.7)$ & $0(0)$ & 0.236 & $50(100)$ & $50(100)$ & $<0.001$ \\
\hline Time-to-operation (h) & & & 0.238 & & & 0.138 \\
\hline$<6$ & $24(8.3)$ & $8(16)$ & & $6(12)$ & $8(16)$ & \\
\hline 6 to 24 & $204(70.3)$ & $32(64)$ & & $35(70)$ & $32(64)$ & \\
\hline$>24$ & $62(21.4)$ & $10(20)$ & & $9(18)$ & $10(20)$ & \\
\hline
\end{tabular}

Values are presented as median (interquartile range), mean \pm standard deviation, or $\mathrm{n}(\%) . S M D$, Standardized mean difference 
TABLE 2. Operative characteristics were similar in propensity matched populations. Continuous variables are presented with standard deviation or interquartile range, for non-normally distributed data. Comparisons were made with Student $t$ test or Wilcoxon rank-sum test, respectively. Categorical variables are reported with absolute counts and percentages, and comparisons were made with the $\chi^{2}$ test or Fisher exact test as necessary

\begin{tabular}{|c|c|c|c|}
\hline \multirow[b]{2}{*}{ Characteristic } & \multicolumn{2}{|c|}{ After matching } & \multirow[b]{2}{*}{$P$ value } \\
\hline & $\frac{\text { No neurologic injury }}{(\mathrm{n}=\mathbf{5 0})}$ & $\frac{\text { Neurologic injury }}{(\mathbf{n}=\mathbf{5 0})}$ & \\
\hline Cardiopulmonary bypass time (min) & $206.22 \pm 63.83$ & $207.86 \pm 63.42$ & .9 \\
\hline Aortic crossclamp time (min) & $126.56 \pm 50.69$ & $126.53 \pm 46.10$ & 1.0 \\
\hline Lowest temperature $\left({ }^{\circ} \mathrm{C}\right)$ & $24.68 \pm 3.31$ & $23.84 \pm 3.25$ & .2 \\
\hline Duration of circulatory arrest (min) & $25.70 \pm 9.59$ & $28.84 \pm 10.84$ & .2 \\
\hline $\begin{array}{l}\text { Cerebral protection strategy } \\
\text { None (operation performed with clamp on) } \\
\text { Selective antegrade cerebral perfusion } \\
\text { Retrograde cerebral perfusion } \\
\text { Deep hypothermic circulatory arrest }\end{array}$ & $\begin{array}{c}8(16.0) \\
36(72.0) \\
2(4.0) \\
4(8.0)\end{array}$ & $\begin{array}{c}7(14.3) \\
38(77.6) \\
0(0.0) \\
4(8.2)\end{array}$ & .6 \\
\hline Intraoperative packed red blood cell & $3(0-4)$ & $2(0-5)$ & .7 \\
\hline Intraoperative fresh frozen plasma & $6(4-10)$ & $6(4-8)$ & .7 \\
\hline Intraoperative platelet & $2(2-3)$ & $2(2-3)$ & .4 \\
\hline Intraoperative cryoprecipitate & $0(0-1.75)$ & $0(0-2)$ & .5 \\
\hline Aortic root replacement & $14(28.0)$ & $11(22.0)$ & .6 \\
\hline Hemiarch reconstruction & $41(82.0)$ & $40(80.0)$ & 1.0 \\
\hline Total arch replacement & $1(2.0)$ & $2(4.0)$ & 1.0 \\
\hline
\end{tabular}

Values are presented as mean \pm standard deviation, median (interquartile range), or $\mathrm{n}(\%)$.

with myocardial infarction. Our 1:1 optimal matching algorithm was able to create balanced groups (Table 1). Operative characteristics were similar after matching (Table 2). Median follow-up for the matched cohort was 1.3 years (interquartile range, 33 days to 4.6 years); the duration of follow-up was heavily influenced by the high perioperative mortality in the neurologic injury group.

\section{Survival}

In the matched analysis, in-hospital mortality was greater for patients presenting with neurologic injury $(22.0 \% ; 11$ of 50) compared with those presenting without neurologic injury ( $6 \% ; 3$ of 50$)$ (Table 3), OR, 4.42 (95\% CI, 1.15$16.97 ; P=.03$ ). In an exploratory analysis, this mortality difference appeared to be driven primarily by patients with persistent neurologic deficits (11 of $31 ; 35.5 \%$ ) compared with patients with transient neurologic injuries (0 of 19; $0 \%)(P=.003)$. For comparison, in-hospital mortality for the unmatched population without neurologic injury was $7.3 \%$ (18 of 245). Midterm survival was no different between matched populations in the Kaplan Meier Analysis (log-rank $P=.1$ ), although the number at risk beyond 6 years was limited (Figure 1).

We further explored the association between degree of neurologic injury and perioperative mortality. Persistent neurologic injury was separated into obtunded/coma $(\mathrm{n}=12)$; altered mental status [verbal GCS $<5](\mathrm{n}=9)$, hemiplegia $(\mathrm{n}=10)$, and other primary presenting neurologic symptom $(n=4)$-notably, 4 patients had both hemiplegia and either altered mental status or obtundation; there were 19 patients presenting with transient neurologic injuries. After variable selection, logistic regression demonstrated that obtundation/coma at presentation (OR, 14.12; 95\% CI, 3.58-55.73; $P<.001)$, shock at presentation (OR, 3.33; 95\% CI, 1.34-8.24; $P=.009$ ), myocardial infarction at presentation (OR, 13.64; 95\% CI, 2.79$66.70 ; P=.001)$, and older age (OR, 1.05 per year; $95 \%$ CI, 1.01-1.08; $P=.006)$ were associated with increased risk of perioperative death (Table 4).

\section{Neurologic Outcome}

For patients presenting with persistent neurologic injuries, complete resolution of symptoms was seen in $35.5 \%$ of patients before discharge (11 of 31 ). Among patients in the transient neurologic deficit group, $5.3 \%$ of patients (1 of 19) experienced postoperative deficits similar to the transient deficit at presentation; $21.1 \%$ (4 of 19) experienced postoperative stroke. Of 50 patients with preoperative neurologic injuries, 21 patients underwent postoperative head imaging with either computed tomography or magnetic resonance imaging. Two patients experienced hemorrhagic complications. Of 29 patients 
TABLE 3. Postoperative outcomes in propensity-matched populations. Only in-hospital mortality differed between the 2 groups

\begin{tabular}{|c|c|c|c|}
\hline \multirow[b]{2}{*}{ Outcome } & \multicolumn{2}{|c|}{ After matching } & \multirow[b]{2}{*}{$P$ value } \\
\hline & $\frac{\text { No neurologic injury }}{(n=50)}$ & $\frac{\text { Neurologic injury }}{(n=50)}$ & \\
\hline In-hospital mortality & $3(6.0)$ & $11(22.0)$ & .04 \\
\hline Atrial fibrillation & $22(44.0)$ & $14(28.0)$ & .1 \\
\hline Intubation $>48 \mathrm{~h}$ & $16(32.0)$ & $22(44.0)$ & .3 \\
\hline Tracheostomy & $1(2.0)$ & $6(12.0)$ & .1 \\
\hline Acute kidney injury & $20(40.0)$ & $18(36.0)$ & .8 \\
\hline Hemodialysis & $6(12.0)$ & $5(10.0)$ & 1.0 \\
\hline Fasciotomy & $2(4.0)$ & $1(2.0)$ & 1.0 \\
\hline Heparin-induced thrombocytopenia and thrombosis & $4(8.0)$ & $3(6.0)$ & 1.0 \\
\hline Mediastinal re-exploration & $7(14.0)$ & $5(10.0)$ & .8 \\
\hline Intensive care unit length of stay (d) & $5(3-9)$ & $4(3-10)$ & .7 \\
\hline Hospital length of stay (d) & $10(8-16)$ & $9.50(5.25-18.75)$ & .3 \\
\hline
\end{tabular}

Values are presented as $\mathrm{n}(\%)$ or median (interquartile range).

who did not undergo postoperative head imaging, 5 patients died. The remaining 24 patients either had resolution or significant improvement of symptoms and so imaging was not indicated.

A histogram of time-to-operation demonstrated that most patients underwent an operation within 24 hours (Figure 2). Among patients with persistent neurologic deficits at presentation, receiver operating characteristic curve analysis suggested that the time between symptoms and the start

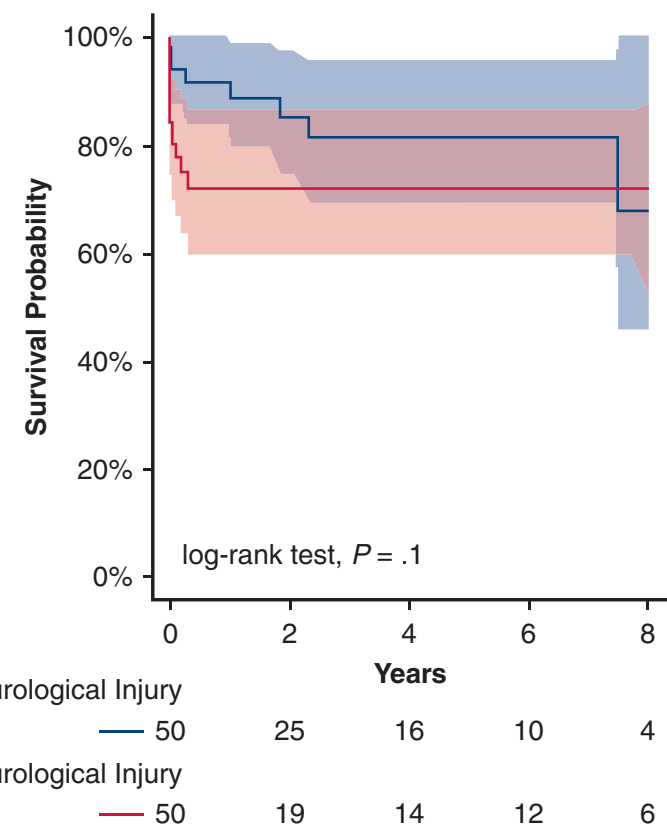

FIGURE 1. Kaplan-Meier survival comparing matched patients presenting with neurologic injury compared with patients who did not. There was a notable early separation in the survival curves, but no significant difference arose at midterm follow-up between the 2 groups, although the number of patients with midterm follow-up was limited. of the operation was a poor predictor of neurologic improvement (cross-validated AUC, 0.40) (Figure 3). There was no threshold for time-to-operation that predicted inhospital mortality (cross-validated AUC, 0.49). In our cohort, there were 11 patients who had an operation beyond the 10-hour threshold suggested by Estrera and colleagues. ${ }^{7}$ Seven of these 11 patients had an improvement in neurologic status following the operation with 3 of 7 patients experiencing complete resolution of their preoperative neurologic deficits.

\section{DISCUSSION}

Neurologic injury complicating acute type A aortic dissection is a challenge to cardiovascular surgeons given the high risk for morbidity and mortality associated with surgery. Previously reported single-center experiences have demonstrated that early operation might lead to superior clinical outcomes, but the upper limit of time-tooperation has not been well defined. In a contemporary cohort at a tertiary referral center, preoperative neurologic injury was associated with an increased risk of in-hospital mortality, and obtundation/coma at presentation was a strong risk factor for perioperative mortality. Transient neurologic injury and hemiplegia did not appear to affect perioperative mortality. No threshold for time-tooperation was predictive of neurologic outcome or perioperative mortality.

\section{Immediate Operative Intervention Is Justified}

Concerns about the potential for hemorrhagic complications with systemic heparinization for cardiopulmonary bypass exist. ${ }^{2}$ However, neither Estrera nor Tsukube and colleagues $^{8}$ reported any hemorrhagic complications in 14 and 21 patients undergoing immediate operation for acute type A aortic dissection complicated by neurologic injury 
TABLE 4. Prediction model for postoperative in-hospital mortality demonstrating that obtundation/coma at presentation was the only significant neurologic predictor of in-hospital mortality after accounting for shock and myocardial infarction at the time of presentation. Neither hemiplegia nor transient neurologic injury was selected as a predictor

\begin{tabular}{|c|c|c|}
\hline $\begin{array}{l}\text { Predictor of in-hospital } \\
\text { mortality }\end{array}$ & $\begin{array}{c}\text { Odds ratio } \\
\text { (95\% confidence } \\
\text { interval) }\end{array}$ & $\begin{array}{c}P \\
\text { value }\end{array}$ \\
\hline Obtunded/coma at presentation & $14.12(3.58-55.73)$ & $<.001$ \\
\hline Shock at presentation & $3.33(1.34-8.24)$ & .009 \\
\hline $\begin{array}{l}\text { Myocardial infarction at } \\
\text { presentation }\end{array}$ & $13.64(2.79-66.70)$ & .001 \\
\hline Age (per year) & $1.05(1.01-1.08)$ & .006 \\
\hline
\end{tabular}

at presentation, respectively. Our report is consistent with these prior findings with only 2 patients experiencing intracranial hemorrhage. Although the risk of intracranial hemorrhage associated with systemic anticoagulation in the setting of neurologic injury must be considered, this complication does not appear to be a common occurrence. Notably, the incidence of symptomatic intracranial hemorrhage after mechanical thrombectomy was $7 \%$ in the DWI or CTP Assessment With Clinical Mismatch in the Triage of Wake-Up and Late Presenting Strokes Undergoing Neurointervention With Trevo (DAWN) trial and 6\% in the Endovascular Therapy Following Imaging Evaluation for Ischemic Stroke (DEFUSE 3) trial. The incidence of hemorrhagic events reported in these trials was no different

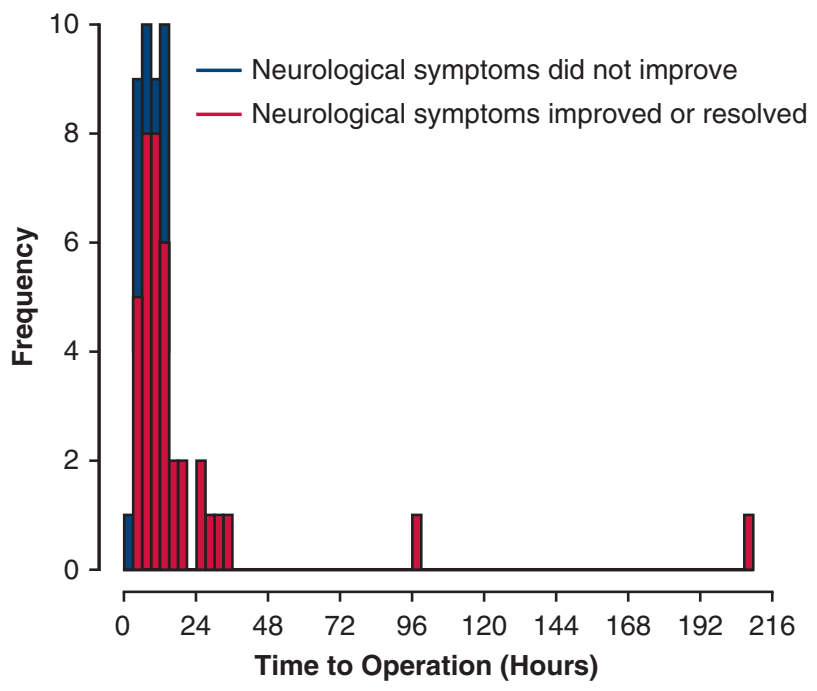

FIGURE 2. Histogram demonstrating the distribution of time-tooperation. The majority of patients arrived in our operating room within 24 hours of the onset of symptoms. Some patients' neurologic symptoms improved or resolved following the operation (red). Some patients' neurologic symptoms did not improve (blue). Many patients who had a prolonged time-to-operation experienced neurologic improvement.

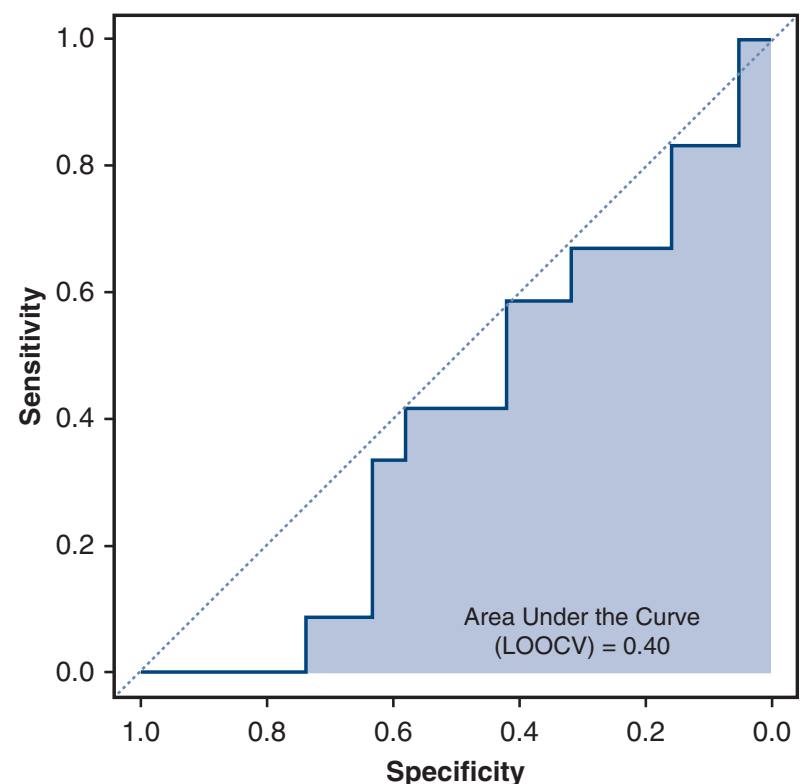

FIGURE 3. Cross-validated receiver operating characteristic curve for predicting lack of neurologic improvement using time-to-operation among patient presenting with neurologic injuries. The area under the curve was 0.40 , suggesting that time-to-operation was a poor predictor of neurologic improvement. LOOCV, Leave-one-out cross-validation.

from the medical management arms (with limited use of intravenous alteplase) of each trial, $4 \%$ and $3 \%$, respectively, suggesting that a proportion of patients with ischemic neurologic insults experience spontaneous hemorrhagic conversion. ${ }^{13,14}$

Both Estrera and colleagues ${ }^{7}$ and Tsukube and colleagues ${ }^{8}$ have previously reported that operations beyond 10 hours or 5 hours, respectively, might be associated with worse outcome. As a result, prolonged time-tooperation might be viewed as a relative contraindication to surgery. This approach was consistent with prior evidence for management of acute ischemic stroke: in a meta-analysis of 5 randomized Phase 3 trials reported in 2016, the upper limit for the benefit of intervention in acute ischemic stroke had been suggested to be 7.3 hours. ${ }^{9}$ However, patients in our cohort regained significant function beyond this window, and time-to-operation did not appear to be predictive of neurologic outcome.

Neurologic injury in acute type A aortic dissection is likely the result of either obstruction of a carotid artery proximally or a low cardiac output state resulting in watershed injury; these may be evolving processes with ongoing ischemia at the time of presentation as opposed to infarcts. The remedy for these patients is to reconstitute antegrade true lumen flow and address the hemodynamic embarrassment. This strategy appears to be supported by more recent randomized trial evidence suggesting that patients suffering from acute ischemic stroke with ongoing ischemic brain tissue benefit from intervention out to 16 to 24 hours. ${ }^{13,14}$ 
This expanded window of opportunity for intervention in patients with neurologic injury complicating the presentation of acute type A aortic dissection is important because many of the patients undergoing repair of acute type A aortic dissection at our institution and in centers participating in the International Registry of Acute Aortic Dissection are referred from other facilities. ${ }^{1,24}$ As a result, patients may frequently experience prolonged periods of time before reperfusion, and these delays may be beyond the thresholds indicated by either Tsukube and colleagues $^{8}$ or Estrera and colleagues. ${ }^{7}$ This introduces a clinical conundrum to cardiac surgeons in the community. It remains unclear whether patients with evolving neurologic injuries should be transferred to a center with greater experience in aortic surgery or undergo immediate operative repair in the hope of preserving brain. The answer will vary depending on patient stability and the availability of both air-ambulance and an accepting aortic referral center.

Given the difficulty in predicting neurologic outcome and the poor results with medical management reported in the International Registry of Acute Aortic Dissection, ${ }^{5}$ our policy is to proceed directly to the operating room for immediate surgery in all patients presenting with acute type A aortic dissection complicated by neurologic injury at presentation, including patients with distal malperfusion syndromes ${ }^{1}$; the only exception is brain death on presentation to our facility. The majority of our patients are transferred from other institutions, ${ }^{1}$ and - to avoid unnecessary operative delay-no additional studies, including computed tomography of the head, are obtained. The diagnosis of acute type A aortic dissection is confirmed by transesophageal echocardiogram in the operating room and by review of the outside study by a surgeon at our institution. Our operative approach involves selective antegrade cerebral perfusion via an axillary artery perfusion limb in most cases; however, direct true lumen cannulation using epiaortic ultrasound has also been used. We typically perform arch repairs under moderate hypothermic circulatory arrest $\left(24^{\circ} \mathrm{C}-28^{\circ} \mathrm{C}\right)$ but will cool further $\left(22^{\circ} \mathrm{C}-24^{\circ} \mathrm{C}\right)$ for total arch replacement using a multibranch graft. Near infrared spectroscopy is routinely used to monitor cerebral oximetry. Trivedi and colleagues ${ }^{25}$ reported excellent outcomes in acute type A aortic dissection employing an aggressive approach that included total arch replacement with additional common carotid replacement. In our experience, total arch replacement is not frequently necessary, and reconstitution of antegrade true lumen flow in the distal aorta and head vessels may be achieved with a peninsula-style transverse arch repair. ${ }^{26}$

\section{Neurologic Injuries Are Not All Created Equal}

Among the limitations influencing prior analyses of neurologic injury in acute type A aortic dissection has been the lack of granularity in differentiating among neurologic injuries. We attempted to distinguish between not only persistent and transient neurologic injuries but also among specific presenting symptoms. Among patients with persistent neurologic injuries, presenting with obtundation/coma was the only deficit associated with increased odds of death; this was the case after accounting for shock, which may have been a major contributor to altered mental status among several patients who presented in extremis. In contrast, transient neurologic injury, hemiplegia, and other deficits failed to be associated with perioperative mortality.

\section{Limitations and Strengths}

This study was limited by its retrospective nature at a high-volume aortic referral center; whether this result is generalizable is uncertain. The existence of a volumeoutcome relationship in proximal aortic surgery may influence whether immediate operation should be undertaken or referral to an aortic referral center should be pursued. ${ }^{27}$ Related to this, the vast majority of patients undergoing an operation at our institution were referred from other centers. Whether delays attributable to transfer or hemodynamic instability during transport may have contributed to perioperative mortality or reduced the volume of salvageable brain tissue is uncertain. However, with an increasing proportion of patients with aortic dissection being transferred from primary centers to aortic referral centers, ${ }^{24}$ this scenario may be the rule rather than the exception.

Despite having a larger number of patients with neurologic injury than prior single-institution studies, our study likely was not large enough and lacked the granularity either to estimate the risk attributable to finer gradations of injury or to evaluate potential prognostic factors; for example, dynamic versus static head vessel occlusion. Future single or multi-institutional studies will be necessary to examine the role of preoperative head imaging in risk stratification and operative planning. A larger multiinstitutional study may also be able to delineate the role of total arch replacement and cerebral protection strategies (eg, moderate vs deep hypothermia and antegrade vs retrograde cerebral perfusion) in these complicated patients. Finally, it may be necessary to further investigate the result of our exploratory analysis regarding the effect of presenting symptoms on postoperative outcome. The unselected nature of the cohort-nearly all patients with neurologic deficits underwent immediate open surgical repair-over a relatively limited period of time represents an important strength of this study because the potential for either treatment selection bias or secular trend was limited.

\section{CONCLUSIONS}

Neurologic injury at the time of presentation with acute type A aortic dissection was associated with an increased risk of in-hospital mortality and obtundation/coma was a 
strong risk factor for perioperative mortality. Among patients with persistent neurologic injuries, no threshold for time-to-operation was predictive of neurologic outcome or perioperative mortality. Given the abysmal outcomes associated with medical management of these patients, prolonged time from onset of symptoms in patients presenting with neurologic injury should not act as a contraindication to operation.

\section{Conflict of Interest Statement}

Dr Fischbein receives funding from the National Institutes of Health (grant No. R01AR066629-01A1). All other authors have nothing to disclose with regard to commercial support.

\section{References}

1. Chiu P, Tsou S, Goldstone AB, Louie M, Woo YJ, Fischbein MP. Immediate operation for acute type A aortic dissection complicated by visceral or peripheral malperfusion. J Thorac Cardiovasc Surg. 2018;156:18-24.e3.

2. Cambria RP, Brewster DC, Gertler J, Moncure AC, Gusberg R, Tilson MD, et al. Vascular complications associated with spontaneous aortic dissection. J Vasc Surg. 1988;7:199-209.

3. Geirsson A, Szeto WY, Pochettino A, McGarvey ML, Keane MG, Woo YJ, et al. Significance of malperfusion syndromes prior to contemporary surgical repair for acute type A dissection: outcomes and need for additional revascularizations. Eur J Cardiothorac Surg. 2007;32:255-62.

4. Bossone E, Corteville DC, Harris KM, Suzuki T, Fattori R, Hutchison S, et al Stroke and outcomes in patients with acute type A aortic dissection. Circulation. 2013;128:S175-9.

5. Di Eusanio M, Patel HJ, Nienaber CA, Montgomery DM, Korach A, Sundt TM et al. Patients with type A acute aortic dissection presenting with major brain injury: should we operate on them? J Thorac Cardiovasc Surg. 2013;145: 213-21.e211.

6. Fann JI, Sarris GE, Miller DC, Mitchell RS, Oyer PE, Stinson EB, et al. Surgical management of acute aortic dissection complicated by stroke. Circulation. 1989; 80:1257-63.

7. Estrera AL, Garami Z, Miller CC, Porat EE, Achouh PE, Dhareshwar J, et al. Acute type A aortic dissection complicated by stroke: can immediate repair be performed safely? J Thorac Cardiovasc Surg. 2006;132:1404-8.

8. Tsukube T, Hayashi T, Kawahira T, Haraguchi T, Matsukawa R, Kozawa S, et al. Neurological outcomes after immediate aortic repair for acute type A aortic dissection complicated by coma. Circulation. 2011;124:S163-7.

9. Saver JL, Goyal M, van der Lugt A, Menon BK, Majoie CB, Dippel DW, et al. Time to treatment with endovascular thrombectomy and outcomes from ischemic stroke: a meta-analysis. JAMA. 2016;316:1279-88.

10. Powers WJ, Derdeyn CP, Biller J, Coffey CS, Hoh BL, Jauch EC, et al. 2015 American Heart Association/American Stroke Association focused update of the 2013 guidelines for the early management of patients with acute ischemic stroke regarding endovascular treatment: a guideline for healthcare professionals from the American Heart Association/American Stroke Association. Stroke. 2015;46:3020-35.

11. Fransen PS, Berkhemer OA, Lingsma HF, Beumer D, van den Berg LA, Yoo AJ, et al. Time to reperfusion and treatment effect for acute ischemic stroke: a randomized clinical trial. JAMA Neurol. 2016;73:190-6.

12. Powers WJ, Rabinstein AA, Ackerson T, Adeoye OM, Bambakidis NC, Becker K, et al. 2018 guidelines for the early management of patients with acute ischemic stroke: a guideline for healthcare professionals from the American Heart Association/American Stroke Association. Stroke. 2018;49:e46-110.

13. Albers GW, Marks MP, Kemp S, Christensen S, Tsai JP, Ortega-Gutierrez S, et al. Thrombectomy for stroke at 6 to 16 hours with selection by perfusion imaging. N Engl J Med. 2018;378:708-18.

14. Nogueira RG, Jadhav AP, Haussen DC, Bonafe A, Budzik RF, Bhuva P, et al. Thrombectomy 6 to 24 hours after stroke with a mismatch between deficit and infarct. $N$ Engl J Med. 2018:378:11-21.

15. Blackstone EH. Demise of a vital resource. J Thorac Cardiovasc Surg. 2012;143: 37-8.

16. Peterss S, Charilaou P, Ziganshin BA, Elefteriades JA. Assessment of survival in retrospective studies: the social security death index is not adequate for estimation. J Thorac Cardiovasc Surg. 2017;153:899-901.

17. Rothman KJ. No adjustments are needed for multiple comparisons. Epidemiology. 1990;1:43-6.

18. Silber JH, Rosenbaum PR, Trudeau ME, Even-Shoshan O, Chen W, Zhang X, et al. Multivariate matching and bias reduction in the surgical outcomes study. Med Care. 2001;39:1048-64.

19. Austin PC, Tu JV. Automated variable selection methods for logistic regression produced unstable models for predicting acute myocardial infarction mortality. J Clin Epidemiol. 2004:57:1138-46.

20. Austin PC, Tu JV. Bootstrap methods for developing predictive models. Am Stat. 2004;58:131-7.

21. Austin PC. Bootstrap model selection had similar performance for selecting authentic and noise variables compared to backward variable elimination: a simulation study. J Clin Epidemiol. 2008;61:1009-117.e1001.

22. Wasserman L, Roeder K. High dimensional variable selection. Ann Stat. 2009; 37:2178-201.

23. Wu J, Devlin B, Ringquist S, Trucco M, Roeder K. Screen and clean: a tool for identifying interactions in genome-wide association studies. Genet Epidemiol. 2010;34:275-85.

24. Pape LA, Awais M, Woznicki EM, Suzuki T, Trimarchi S, Evangelista A, et al. Presentation, diagnosis, and outcomes of acute aortic dissection: 17-year trends from the international registry of acute aortic dissection. J Am Coll Cardiol. 2015;66:350-8

25. Trivedi D, Navid F, Balzer JR, Joshi R, Lacomis JM, Jovin TG, et al. Aggressive aortic arch and carotid replacement strategy for type A aortic dissection improves neurologic outcomes. Ann Thorac Surg. 2016;101:896-903.

26. Itoh A, Fischbein M, Arata K, Miller DC. "Peninsula-style" transverse aortic arch replacement in patients with bicuspid aortic valve. Ann Thorac Surg. 2010;90:1369-71.

27. Hughes GC, Zhao Y, Rankin JS, Scarborough JE, O'Brien S, Bavaria JE, et al. Effects of institutional volumes on operative outcomes for aortic root replacement in North America. J Thorac Cardiovasc Surg. 2013;145:166-70.

Key Words: stroke, neurologic injury, aortic dissection 